\title{
A-Posteriori Error Analysis of a Mixed Method for Linear Parabolic Problem
}

\author{
M.I. Asensio, J.M. Cascón, and L. Ferragut \\ Departamento de Matemática Aplicada, Universidad de Salamanca, \\ 37008 Salamanca, Spain \\ \{mas, casbar, ferragut\}@usal.es
}

\begin{abstract}
In this paper we present a-posteriori error estimator for the mixed formulation of linear parabolic problem, and we use them in designing an efficient adaptive algorithm. Our space-time discretization consist of lowest order Raviart-Thomas finite element over graded meshes, and discontinuous Galerkin method with varying time-steps.
\end{abstract}

\section{Introduction}

A-posteriori error estimates are an essential component in the design of reliable and efficient adaptive algorithms for the numerical solutions of PDEs. At the same time, mixed formulations are suitable for certain problems, as they allow to directly approach certain derivatives of the solution.

In this paper we introduce an a-posteriori error estimation for mixed formulation of the linear parabolic problem obtaining,

$$
\|u-U\|_{L^{\infty}\left(0, T ; L^{2}(\Omega)\right)}+\|\mathbf{p}-\mathbf{P}\|_{L^{2}\left(0, T ; H^{-1}(\mathrm{div}, \Omega)\right)} \leq \mathcal{E}\left(u_{0}, f, T, \Omega ; U, \mathbf{P}, h, k\right),
$$

where $u$ is the scalar variable, and $\mathbf{p}$ its gradient. Capital letters represent the numerical approximations obtained with the lowest order Raviart-Thomas finite element in space and a variable step discontinuous Galerkin method in time. The estimator $\mathcal{E}$ is computable in terms of: given data $u_{0}, f, \Omega, T$, computed solutions $U$ and $\mathbf{P}$, meshsize $h$ and time step $k$.

As in [4], we obtain the error representation formulas of $u-U$ and $\mathbf{p}-\mathbf{P}$ from the residual equations. The evaluation of the residual in the corresponding norms and the stability properties of the associated dual problem allow the estimations to be concluded. The scalar error bound is an extension to the mixed formulation of the results developed in 3 . For the error estimation of $\mathbf{p}-\mathbf{P}$ we use the Helmholtz descomposition in $L^{2}\left(\Omega ; \mathbb{R}^{2}\right)$, as in [1] and [2] for the stationary case.

\section{Continuous Problem}

Let $\Omega \subset \mathbb{R}^{2}$ be a bounded convex polyhedral domain, let $T>0$ be the final time, and set $Q_{T}:=\Omega \times(0, T)$ and $\Gamma_{T}:=\partial \Omega \times(0, T)$. Given an initial condition 
$u_{0}$ and a source term $f(x, t)$, let $(u, \mathbf{p})$ be the solution of the problem,

$$
\begin{array}{rll}
u_{t}+\operatorname{div} \mathbf{p}=f & \text { in } & Q_{T}, \\
\mathbf{p}+\nabla u=0 & \text { in } & Q_{T}, \\
\nabla u \cdot \boldsymbol{\nu}=0 & \text { on } & \Gamma_{T}, \\
u(., 0)=u_{0} & \text { in } & \Omega,
\end{array}
$$

where $\boldsymbol{\nu}$ is the unit normal vector to $\partial \Omega$.

In the following, $(\cdot, \cdot)$ denotes the inner product in $L^{2}(\Omega)$ or $L^{2}\left(\Omega ; \mathbb{R}^{2}\right)$ and $<\cdot, \cdot>$ denotes the duality pairing between the corresponding spaces. We define the space $H_{0}(\operatorname{div}, \Omega)$ as,

$$
H_{0}(\operatorname{div}, \Omega):=\left\{\mathbf{q} \in L^{2}\left(\Omega ; \mathbb{R}^{2}\right): \operatorname{div} \mathbf{q} \in L^{2}(\Omega), \quad<\mathbf{q} \cdot \boldsymbol{\nu}, v>=0 \forall v \in H^{1}(\Omega)\right\}
$$

and denote by $H^{-1}(\operatorname{div}, \Omega)$ its dual space. For the corresponding variational formulation of this problem we use the bilinear operator $B$, defined on $L^{2}(\Omega) \times$ $H_{0}(\operatorname{div}, \Omega)$ as,

$$
\mathrm{B}(w, \mathbf{q} ; r, \mathbf{s})=(w, r)+(\operatorname{div} \mathbf{q}, r)+(\operatorname{div} \mathbf{s}, w)-(\mathbf{q}, \mathbf{s}) .
$$

Adding the equations (1) and (2) we obtain the following variational problem,

Continuous problem. Find $u$ and $\mathbf{p}$ such that

$$
\begin{gathered}
u \in H^{1}\left(0, T ; L^{2}(\Omega)\right), \quad \mathbf{p} \in L^{2}\left(0, T ; H_{0}(\operatorname{div}, \Omega)\right), \quad u(., 0)=u_{0}, \\
\mathrm{~B}\left(u_{t}, \mathbf{p} ; v, \mathbf{q}\right)=(f, v) \quad \forall(v, \mathbf{q}) \in L^{2}(\Omega) \times H_{0}(\operatorname{div}, \Omega) \text { a.e. } t \in(0, T) .
\end{gathered}
$$

\section{Discretization}

The fully discrete problem combines finite elements in space with the Galerkin discontinuous method in time.

Let $k_{n}$ denotes the time step at the $n$-th step and set $t_{n}=\sum_{i=1}^{n} k_{i}$. Let $N$ be the total number of time steps, that is, $t_{N}=T$. To each time step, $\left(t_{n-1}, t_{n}\right]$ we associate $\left(\mathcal{T}_{n}, \mathbb{M}_{n}, \mathbb{X}_{n}\right)$, where $\mathcal{T}_{n}=\left\{\mathcal{S}_{n}\right\}$ is a uniformly regular partition of $\Omega$ in triangles. We denote $h_{n}$ a piecewise constant function with $h_{n \mid \mathcal{S}}=\operatorname{diam}(\mathcal{S})$ for all $\mathcal{S} \in \mathcal{T}_{n}, \mathcal{B}_{n}$ is the set of all interior edges $e$ of $\mathcal{T}_{n} ; h_{e}$ stands for the size of $e$. The mesh $\mathcal{T}_{n}$ is obtained from $\mathcal{T}_{n-1}$ by refining/coarsening. $\mathbb{M}_{n} \in L^{2}(\Omega)$ is the usual space of piecewise constant finite elements over $\mathcal{T}_{n}$. Finally, $\mathbb{X}_{n} \in H(\operatorname{div}, \Omega)$ is the lowest order Raviart-Thomas finite element. Then, we have the problem, Discrete problem. Given $U^{n-1} \in \mathbb{M}_{n-1}, \mathbf{P}^{n-1} \in \mathbb{X}_{n-1}$, then $k_{n-1}$ and $\mathcal{T}_{n-1}$ are modified as described in section 5 to get $k_{n}$ and $\mathcal{T}_{n}$ and thereafter $U^{n} \in \mathbb{M}_{n}, \mathbf{P}^{n} \in \mathbb{X}_{n}$ computed according to,

$$
\begin{gathered}
\frac{1}{k_{n}}\left(U^{n}-\pi_{\Omega} U^{n-1}, V\right)+\left(\operatorname{div} \mathbf{P}^{n}, V\right)=\frac{1}{k_{n}} \int_{t_{n-1}}^{t_{n}}(f, V) d t, \quad \forall V \in \mathbb{M}_{n}, \\
\left(\mathbf{P}^{n}, \mathbf{Q}\right)-\left(\operatorname{div} \mathbf{Q}, U^{n}\right)=0, \quad \forall \mathbf{Q} \in \mathbb{X}_{n},
\end{gathered}
$$

where $\pi_{\Omega}^{n}$ denotes the usual $L^{2}(\Omega)$-projection operator over $\mathbb{M}_{n}$. 


\section{A-Posteriori Estimator}

In the following Theorems we present the a-posteriori error estimates for the schemes developed bellow.

Theorem 1. Let $(u, \mathbf{p})$ be the solution of (5,6) and $(U, \mathbf{P})$ the solution of (7.8). If $\Omega$ is convex, for $N \geq 1$,

$$
\left\|e_{u}^{t_{N}}\right\|_{L^{2}(\Omega)} \leq\left\|e_{u}^{0}\right\|_{L^{2}(\Omega)}+\sum_{i=1}^{5} C_{i} \max _{1 \leq n \leq N} \mathcal{E}_{i},
$$

with,

$$
\begin{array}{ll}
\mathcal{E}_{1}:=\left\|U^{n}-\pi_{\Omega}^{n} U^{n-1}\right\|_{L^{2}(\Omega)} & \left.\mathcal{E}_{2}:=\| U^{n-1}-\pi_{\Omega}^{n} U^{n-1}\right) \|_{L^{2}(\Omega)}, \\
\mathcal{E}_{3}:=\left\|h_{n} \mathbf{P}\right\|_{L^{2}(\Omega)}, & \mathcal{E}_{4}:=\max _{t \in\left[t_{n-1}, t_{n}\right)}\left\|h_{n}(f-\operatorname{div} \mathbf{P})\right\|_{L^{2}(\Omega)}, \\
\mathcal{E}_{5}:=\int_{t_{n-1}}^{t_{n}}\|f-\operatorname{div} \mathbf{P}\|_{L^{2}(\Omega)} d t . &
\end{array}
$$

Theorem 2. Let $(u, \mathbf{p})$ be the solution of (5,6) and $(U, \mathbf{P})$ the solution of (7.8). Let $J_{\boldsymbol{\tau}}$ be the tangential jump of $\mathbf{P} \in \mathbb{X}$ along e. If $\Omega$ is convex, for $N \geq 1$,

$$
\left\|\mathbf{E}_{\mathbf{P}}\right\|_{L^{2}\left(0, T ; H^{-1}(d i v, \Omega)\right)} \leq\left\|e_{u}^{0}\right\|_{L^{2}(\Omega)}+\sum_{i=6}^{11} C_{i} \mathcal{E}_{i},
$$

where,

$$
\begin{aligned}
& \mathcal{E}_{6}:=\left(\sum_{n=1}^{N} k_{n} \sum_{e \in \mathcal{B}_{n}} h_{e}\left\|J_{\boldsymbol{\tau}}\right\|_{L^{2}(e)}^{2}\right)^{\frac{1}{2}}, \mathcal{E}_{7}:=\left(\sum_{n=1}^{N} k_{n}\left\|U^{n}-\pi_{\Omega} U^{n-1}\right\|_{L^{2}(\Omega)}^{2}\right)^{\frac{1}{2}}, \\
& \mathcal{E}_{8}:=\left(\sum_{n=1}^{N} k_{n}\left\|U^{n-1}-\pi_{\Omega} U^{n-1}\right\|_{L^{2}(\Omega)}^{2}\right)^{\frac{1}{2}}, \mathcal{E}_{9}:=\left(\sum_{n=1}^{N} \int_{t_{n-1}}^{t_{n}}\left\|h_{n}(f-\operatorname{div} \mathbf{P})\right\|_{L^{2}(\Omega)}^{2}\right)^{\frac{1}{2}}, \\
& \mathcal{E}_{10}:=\left[\sum_{n=1}^{N}\left(\int_{t_{n-1}}^{t_{n}} k_{n}^{1 / 2}\|f-\operatorname{div} \mathbf{P}\|_{L^{2}(\Omega)}\right)^{2}\right]^{\frac{1}{2}}, \mathcal{E}_{11}:=\left(\sum_{n=1}^{N} k_{n}\left\|h_{n} \mathbf{P}\right\|_{L^{2}(\Omega)}^{2}\right)^{\frac{1}{2}} .
\end{aligned}
$$

\section{Adaptive Algorithm and Numerical Examples}

We propose two adaptive strategies based on the former estimates. The goal is to determine the time steps and the meshes in order to obtain a uniform error distribution. As usually in parabolic problem we distinguish four kinds of terms in the a-posteriori estimate: initial error, space discretization error, coarsening error and time discretization error,

$$
\mathcal{E}_{n} \leq \mathcal{E}_{0}+\mathcal{E}_{h, n}+\mathcal{E}_{c, n}+\mathcal{E}_{k, n}
$$


We also select the parameters $\Gamma_{0}, \Gamma_{h}, \Gamma_{k}$, verifying, $\Gamma_{0}+\Gamma_{h}+\Gamma_{k} \leq 1$, in a way that given a total error tolerance $t o l$, the adaptive algorithm tries to select time steps and meshes verifying for all $n$,

$$
\mathcal{E}_{n} \sim \text { tol }, \quad \mathcal{E}_{0} \sim \Gamma_{0} \text { tol }, \quad \mathcal{E}_{h, n}+\mathcal{E}_{c, n} \sim \Gamma_{h} \text { tol, } \quad \mathcal{E}_{k, n} \sim \Gamma_{k} \text { tol } .
$$

The algorithm begins with an initial time step $k_{0}$. Given $\theta_{1} \in(0,1)$ and $\theta_{2} \in$ $\left(0, \theta_{1}\right)$, if $\mathcal{E}_{k, n}>\Gamma_{k}$ tol, then the time step diminishes in a factor $\delta_{1} \in(0,1)$; on the contrary, if $\mathcal{E}_{k}<\Gamma_{k}$ tol, the time step increases in a factor $\delta_{2}>1$. For the space refinement we use an error equidistribution strategy (see [3]).

We use an implicit adaptive strategy, that is for each time step we start from the previous step mesh and repeat the process solving-estimating-adapting until the estimated error being below the tolerance.

In the poster session we will present several examples showing that the proposed method is efficient and reliable. We compare the estimators with the exact errors, and we obtain an excelent correlation coefficient number $(\approx 1)$. We also study the adaptation in time and in space.

The numerical experiments have been designed with the finite element toolbox ALBERT [5], extended with new designed tools for the lowest order RaviartThomas finite element and the a-posteriori error estimator developed in this paper.

\section{Conclusions}

We obtain a-posteriori error estimators for the mixed formulation of linear parabolic problem and we use them in designing an efficient adaptive algorithm.

Acknowledgements. Research partially supported by REN2001-0925-03-03, Ministerio de Ciencia y Tecnología (Spain) and SA089/01, Junta de Castilla y León (Spain).

\section{References}

1. A. Alonso, Error estimators for a mixed method, Numer. Math., Vol. 74 (1994), $\mathrm{n}^{0}$ 4, pp. 385-395.

2. C. Cartensen, A posteriori error estimate for mixed finite element method, Math. Comp., Vol. 66 (1997), $\mathrm{n}^{0}$ 218, pp. 465-776.

3. K. Eriksson and C. Johnson, Adaptive Finite Element Methods For Parabolic Problems I: A Linear Model Problem, SIAM J. Numer. Anal., Vol. 28 (1991), $\mathrm{n}^{0}$ 1, pp. 43-77.

4. R. H. Nochetto, A. Schmidt, and C.Verdi, A posterioi error estimation and adaptivity for degenerate parabolic problems, Math. Comp., Vol. 69, (2000), $\mathrm{n}^{0}$ 229, pp. 1-24.

5. A. Schmidt and K.G. Siebert, ALBERT: An adaptive hierarchical finite element toolbox, Preprint 06/2000, Freiburg (2000). 